\title{
A fast method for Stokes profile synthesis
}

\section{Radiative transfer modeling for ZDI and Stokes profile inversion}

\author{
T. A. Carroll, M. Kopf, and K. G. Strassmeier
}

\begin{abstract}
Astrophysikalisches Institut Potsdam, An der Sternwarte 16, 14482 Potsdam, Germany
\end{abstract}
e-mail: tcarroll@aip.de

Received 15 April 2008 / Accepted 12 June 2008

\section{ABSTRACT}

\begin{abstract}
Context. The major challenges for a fully polarized radiative transfer driven approach to Zeeman-Doppler imaging are still the enormous computational requirements. In every cycle of the iterative interplay between the forward process (spectral synthesis) and the inverse process (derivative based optimization) the Stokes profile synthesis requires several thousand evaluations of the polarized radiative transfer equation for a given stellar surface model.

Aims. To cope with these computational demands and to allow for the incorporation of a full Stokes profile synthesis into Dopplerand Zeeman-Doppler imaging applications as well as into large scale solar Stokes profile inversions, we present a novel fast and accurate synthesis method for calculating local Stokes profiles.

Methods. Our approach is based on artificial neural network models, which we use to approximate the complex non-linear mapping between the most important atmospheric parameters and the corresponding Stokes profiles. A number of specialized artificial neural networks, are used to model the functional relation between the model atmosphere, magnetic field strength, field inclination, and field azimuth, on one hand and the individual components $(I, Q, U, V)$ of the Stokes profiles, on the other hand.

Results. We performed an extensive statistical evaluation and show that our new approach yields accurate local as well as diskintegrated Stokes profiles over a wide range of atmospheric conditions. The mean rms errors for the Stokes $I$ and $V$ profiles are well below $0.2 \%$ compared to the exact numerical solution. Errors for Stokes $Q$ and $U$ are in the range of $1 \%$. Our approach does not only offer an accurate approximation to the LTE polarized radiative transfer it, moreover, accelerates the synthesis by a factor of more than 1000 .
\end{abstract}

Key words. line: formation - line: profiles - polarization - radiative transfer - stars: magnetic fields - Sun: magnetic fields

\section{Introduction}

The successful application of Doppler- and Zeeman-Doppler imaging (DI \& ZDI) in the last two decades has enormously contributed to our current knowledge about stellar surface activity and magnetism (Strassmeier \& Rice 1998; Donati 1999; Piskunov \& Kochukhov 2002; Strassmeier 2002; Donati et al. 2003; Kochukhov et al. 2004; Donati et al. 2006). Though it has its limitation, Zeeman-Doppler imaging is the only available method that allows to estimate the surface distribution of stellar magnetic fields. Doppler- and Zeeman-Doppler imaging rely on the complex and computational expensive interplay between the forward process of spectral line synthesis on the one hand and the inverse process of a regularized optimization or fitting procedure on the other hand. Based on a highly discretized surface model Doppler- and Zeeman-Doppler imaging iteratively seeks the best possible solution of the surface temperature and/or magnetic field surface distribution, which is compatible with the observed rotationally-modulated line profiles.

The spectral synthesis plays a decisive role here and requires a good knowledge of accurate atomic data, continuous as well as line opacities, broadening mechanisms, model atmospheres etc. The usual way to cope with the synthesis in DI applications, which allows for the calculation of disk-integrated line profiles in a reasonable time frame, is the compilation of a precalculated database of local line profiles for large number of different atmospheric models and lines-of-sight (LOS) (Rice \& Strassmeier 2000). But unlike DI, the radiative transfer in magnetized media requires the solution of the coupled set of equation for all four Stokes parameters (Rees \& Murphy 1987) and moreover, since the magnetic field is a vector quantity, a proper precalculation quickly becomes prohibitive for ZDI applications in the general case where no simple global magnetic field organization is present.

Due to these complexities and enormous demands many applications of ZDI (but also DI) still rely on approximate methods or even completely avoid a radiative transfer modeling approach. Some use an ad-hoc line synthesis where Stokes $I$ profiles are used from template stars or modeled by simple Gaussian profiles (Donati \& Collier Cameron 1997), from which the corresponding Stokes $V$ profiles are derived by means of the weakfield approximation (Donati \& Brown 1997; Donati et al. 1999). In this respect, one is able to entirely bypass radiative transfer modeling.

In an effort to provide the necessary tools for a fully polarized radiative transfer driven approach to ZDI as well as to provide the diagnostic capabilities for the next generation of a high-resolution spectropolarimeter (PEPSI) at the $8.4 \mathrm{~m}$ Large Binocular Telescope (LBT) (Strassmeier et al. 2003, 2007), we developed the Zeeman-Doppler imaging code iMap (Carroll et al. 2007). To accelerate the synthesis process, our ZDI code incorporates, besides the conventional numerical implementation of the polarized radiative transfer, a novel fast and accurate method for calculating local and disk-integrated synthetic Stokes profiles under local thermodynamic equilibrium (LTE). 
This approach uses artificial neural networks (ANNs) to approximate the polarized radiative transfer calculations. Artificial neural networks have already proven to be a fast alternative for solar Stokes profile inversions and Zeeman tomography (Carroll \& Staude 2001; Socas-Navarro 2005a, 2005b; Lites et al. 2007; Carroll \& Kopf 2008). In the here presented approach ANNs are trained on the basis of the numerical solution of the polarized radiative transfer equation to find an approximate representation of the underlying complex mapping between atmospheric quantities like temperature, magnetic field, velocity, etc., and the corresponding Stokes profiles. A detailed assessment and evaluation of the ANN synthesis confirms the accuracy of our novel approach and moreover demonstrates that the ANN approach can accelerate the synthesis process by three orders of magnitude.

This paper is organized as follows: Since our method strongly depends on the accuracy of the underlying numerical method used for the calculation of synthetic Stokes spectra, we first perform an in-depth analysis and assessment of our polarized radiative transfer code in Sect. 2. For this purpose, we made a detailed comparison between our code iMap and the COSSAM synthesis code (Stift 2000), which was also part of the benchmark test and inter-agreement analysis of Wade et al. (2001). In Sect. 3, we give a short introduction into the ANN models we used in this work and describe the calculation of the training database for different atmospheric models, magnetic field configurations and Zeeman sensitive spectral lines. In Sect. 4 we evaluate the accuracy and performance of the trained ANNs relative to the exact numerical solution. Section 4.2 presents a benchmark test that demonstrates the exceptional speed of the new synthesis method compared to the conventional numerical procedure. Finally, we conclude in Sect. 6 with a discussion and summary of the presented fast Stokes profile synthesis.

\section{Numerical polarized radiative transfer}

Because of the particular importance of an accurate Stokes profile synthesis for this work, we first give a brief overview of the basics of the polarized line formation under local thermal equilibrium (LTE) as well as a short introduction of the polarized radiative transfer module implemented within our ZDI code iMap. This is followed by a thorough benchmark test with the existing polarized radiative transfer code COSSAM (Stift 2000).

\subsection{The transport equation}

The basic transport equation for the Stokes vector $\boldsymbol{I}$ in the frequency $v$ reads,

$\mu \frac{\mathrm{d} \boldsymbol{I}_{v}}{\mathrm{~d} z}=-\mathbf{K}_{v}\left(\boldsymbol{I}_{v}-\boldsymbol{S}_{v}\right)$

where $\mu \equiv \cos (\theta)$ and theta the angle between the surface normal and the LOS. $\boldsymbol{S}_{v}$ is the source vector and $\mathbf{K}_{v}$ the propagation matrix, which contains contributions from true absorption and anomalous dispersion. For the sake of a better readability, we omit the index for the frequency in the following. Assuming local thermodynamic equilibrium (LTE) and neglecting continuum polarization the source vector is given by

$\boldsymbol{S}=B(T) \boldsymbol{e}_{0}$,

where $B(T)$ is the Planck function at local temperature $T$ and $\boldsymbol{e}_{0}=(1,0,0,0)^{T}$. The total absorption or propagation matrix is given by

$\mathbf{K}=\kappa_{\mathrm{c}} 11+\kappa_{0} \boldsymbol{\Phi}$.
Here, $\kappa_{\mathrm{c}}$ is the continuum absorption coefficient, $\kappa_{0}$ the line absorption coefficient, 1 denotes the $4 \times 4$ identity matrix, and $\Phi$ the line propagation matrix, given as

$\boldsymbol{\Phi}=\left(\begin{array}{cccc}\phi_{I} & \phi_{Q} & \phi_{U} & \phi_{V} \\ \phi_{Q} & \phi_{I} & \phi_{V}^{\prime} & -\phi_{U}^{\prime} \\ \phi_{U} & -\phi_{V}^{\prime} & \phi_{I} & \phi_{Q}^{\prime} \\ \phi_{V} & \phi_{U}^{\prime} & -\phi_{Q}^{\prime} & \phi_{I}\end{array}\right)$

where

$\phi_{I}=\frac{1}{2} \phi_{p} \sin ^{2} \gamma+\frac{1}{4}\left(\phi_{r}+\phi_{b}\right)\left(1+\cos ^{2} \gamma\right)$

$\phi_{Q}=\frac{1}{2}\left[\phi_{p}-\frac{1}{2}\left(\phi_{r}+\phi_{b}\right)\right] \sin ^{2} \gamma \cos 2 \chi$

$\phi_{U}=\frac{1}{2}\left[\phi_{p}-\frac{1}{2}\left(\phi_{r}+\phi_{b}\right)\right] \sin ^{2} \gamma \sin 2 \chi$

$\phi_{V}=\frac{1}{2}\left(\phi_{r}-\phi_{b}\right) \cos \gamma$

$\phi_{Q}^{\prime}=\frac{1}{2}\left[\phi_{p}^{\prime}-\frac{1}{2}\left(\phi_{r}^{\prime}+\phi_{b}^{\prime}\right)\right] \sin ^{2} \gamma \cos 2 \chi$

$\phi_{U}^{\prime}=\frac{1}{2}\left[\phi_{p}^{\prime}-\frac{1}{2}\left(\phi_{r}^{\prime}+\phi_{b}^{\prime}\right)\right] \sin ^{2} \gamma \sin 2 \chi$

$\phi_{V}^{\prime}=\frac{1}{2}\left(\phi_{r}^{\prime}-\phi_{b}^{\prime}\right) \cos \gamma$

The inclination angle of the magnetic field vector relative to the LOS is denoted herein as $\gamma$ and the azimuthal angle of the field vector as $\chi$. The absorption profiles $\phi_{p, b, r}$ and the anomalous dispersion profiles $\phi_{p, b, r}^{\prime}$ are given by the Voigt- and Faraday-Voigt function respectively (see, e.g., Rees \& Murphy 1987) and are weighted by the normalized strength of the respective atomic transitions (see, e.g., Stenflo 1994).

\subsection{Physical foundations}

Our LTE code (the forward module of $i$ Map) works on the basis of a given model atmosphere, e.g., provided by ATLAS9 (Kurucz 1993) or Phoenix (Hauschildt et al. 1999) or by semi-empirical models (Holweger \& Mueller 1974). The program reads atomic line parameters, which are extracted from the Vald database (Piskunov et al. 1995; Kupka et al. 1999). The calculations of the continuous opacities rest on a code written by Wittmann (1974) which accounts for free-free and bound-free transitions of $\mathrm{H}^{-}, \mathrm{HI}, \mathrm{He}, \mathrm{He}^{-}, \mathrm{H}_{2}^{-}, \mathrm{H}_{2}^{+}$as well as for the metals $\mathrm{C}, \mathrm{Na}$, $\mathrm{Mg}$. Scattering coefficients are computed for Rayleigh scattering by neutral hydrogen, molecular hydrogen, and neutral helium, and for Thomson scattering by free electrons. Radiation damping and Stark broadening are taken into account as well as collisional broadening (van der Waals) according to Anstee \& O'Mara (1995). Partition functions are calculated according to Bolton (1970) and Aller \& Everett (1972). Abundances, ionization energies, and partition functions are supplied for 83 elements (Wittmann 1974). The Zeeman patterns are calculated under $\boldsymbol{L}-\boldsymbol{S}$ coupling. Voigt- and Faraday-Voigt functions are evaluated using the rational approximation method of Humlicek (1982).

The numerical integration of the polarized radiative transfer is provided by a diagonal element lambda operator (DELO) method (Rees et al. 1989) and accounts for magneto-optical effects. The method is implemented in its linear (original) form as well as in the quadratic version, which uses a parabolic approximation of the source function (Socas-Navarro et al. 2000). 

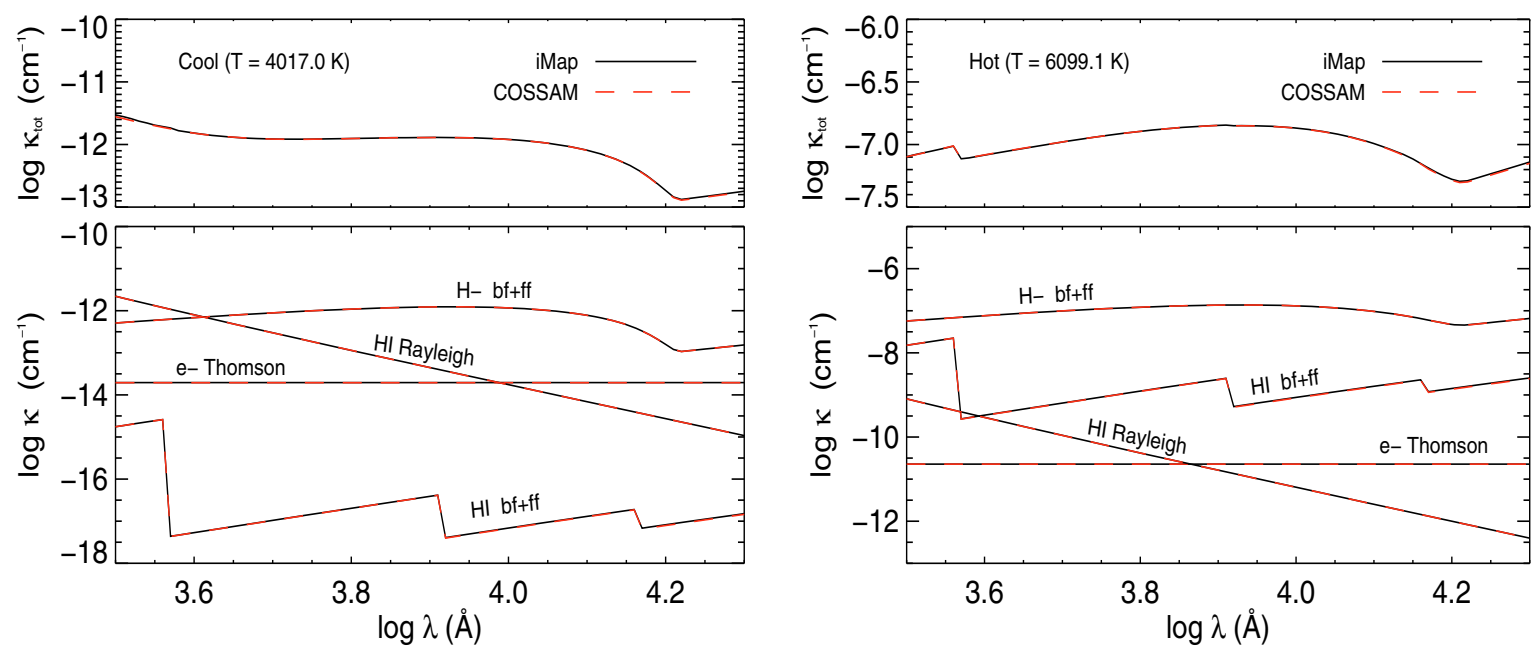

Fig. 1. Total continuous absorption coefficient over the wavelengths (top) calculated with iMap (solid black line) and COSSAM (dashed red line). On the left side we see the calculation for the cold temperature regime and, on the right, for the hot temperature regime. In the bottom panel we see the absorption for the major contributors. Both codes show a very good agreement with rms error smaller than $1 \%$.

For the disk-integration and calculation of stellar (flux) spectra, the underlying surface model in iMap is parameterized on a variable equal-area or equal-degree partition with a minimum element (pixel) size of $1 \times 1$ degree. For each surface element, a local Stokes vector is calculated with respect to its position and underlying atmospheric parameters (abundance, Doppler velocity, bulk velocity, temperature and pressure structures, magnetic field, micro- and macroturbulence). All atmospheric parameters are also allowed to vary along their local vertical direction, which facilitates the complete description of a 3-dimensional atmosphere. Limb darkening is fully accounted for by adjusting the depth stratification of the model atmosphere (temperature and pressure) for each surface element with respect to the LOS, i.e., recalculation of the optical depth scale according to $\mu$ (angle between the local normal vector and the LOS). Field structures on the surface (in temperature, abundance and magnetic fields) can either be described by setting individual surface elements or using spherical harmonics.

\subsection{Interagreement between iMap and COSSAM}

For this benchmark test we follow the framework of Wade et al. (2001), who performed a detailed analysis of three different polarized radiative transfer codes: COSSAM (Stift 2000); INVERS10 (Piskunov \& Kochukhov 2002); ZEEMAN2 (Landstreet 1988). Our goal in this section is to assess the agreement between iMap and COSSAM and by this indirectly also the agreement between iMap and the other synthesis codes of the benchmark test of Wade et al. (2001).

\subsubsection{Continuous opacities}

We first compare the continuous opacities and its variations over wavelength for different temperature regimes. We therefore calculated the continuous opacities for two different temperatures in the range of $\log (\lambda)=3.5$ to $\log (\lambda)=4.3$. We used the Kurucz solar model atmosphere asun (Kurucz 1992) to calculate the total absorption coefficient at $4017 \mathrm{~K}$ and $6099 \mathrm{~K}$ which corresponds to a height of $\log \left(\rho_{x}\right)=-2.185$ and $\log \left(\rho_{x}\right)=$ 0.560 respectively. In Fig. 1 , we have plotted the resulting total continuous absorption coefficients as calculated from both codes, COSSAM and iMap. Additionally, we have plotted the

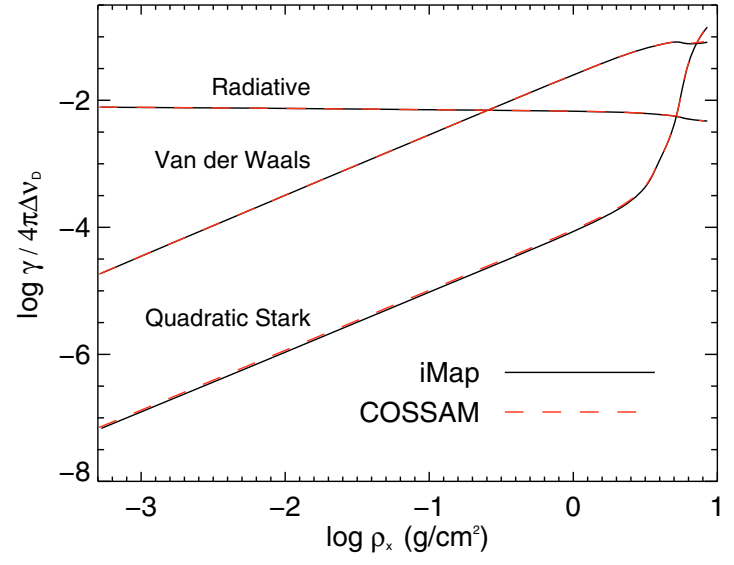

Fig. 2. Radiative, van-der-Waals, and quadratic Stark effect calculated with both codes under the asun atmosphere of Kurucz (1992) (solid black iMap, dashed red COSSAM). For all three contributions to the damping factor the two codes exhibit a very good agreement.

individual main contributors to the continuum absorption (bound-free and free-free for $\mathrm{H}^{-}$and $\mathrm{HI}$, Rayleigh and Thompson scattering). Both synthesis codes show a very good agreement in the continuous absorption coefficients as well as for the individual contributors over the entire wavelength range. The relative rms error is less than $1 \%$.

\subsubsection{Line opacities}

As line opacities are related to the total absorption and the individual absorption profiles, we now turn our attention to the individual damping factors which enters into the absorption and anomalous dispersion profiles, i.e., Voigt- and Faraday-Voigt functions. We follow the investigation of Wade et al. (2001) and compare the radiative, van-der-Waals, and quadratic Stark damping factors. These are calculated again within the asun atmosphere of Kurucz (1992). In Fig. 2, we see the run of the three damping factors with $\log \left(\rho_{x}\right)$. We find a good agreement between the two codes for all three damping contributors with a rms error of less then $0.1 \%$ for the radiative damping term, $0.6 \%$ 


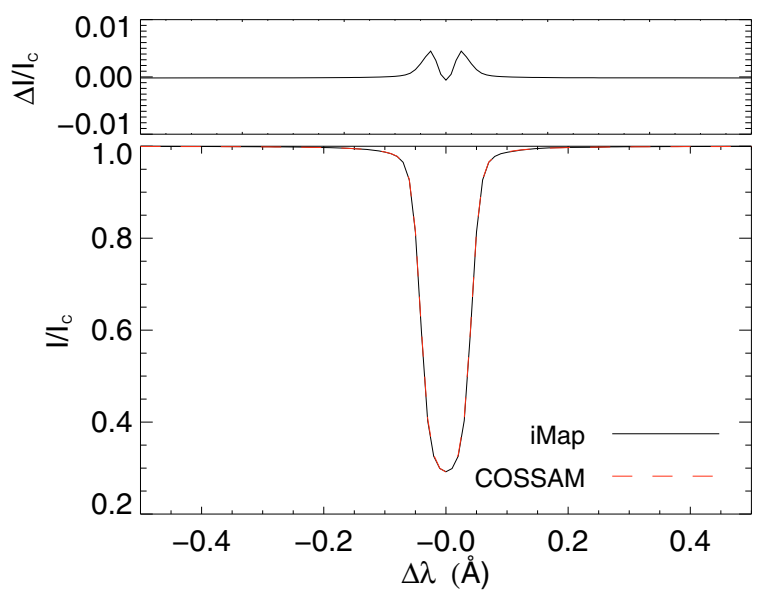

Fig. 3. Comparison of Stokes $I$ profiles for the zero field case (solid black $i$ Map, dashed red COSSAM). Both profile calculations show a very good agreement with an rms error of less than $0.1 \%$. The differences in the profiles are hardly recognizable in the lower plot. The top panel shows the differences $\Delta I / I_{\mathrm{c}}$ between both profiles over the wavelength.

for the van-der-Waals term, and less then $2 \%$ for the quadratic Stark effect.

\subsubsection{Local line profiles - zero magnetic field}

In this section, we compare the combined effect of all physical input parameters and compare directly the resulting line profiles. For this purpose, we synthesized the local line profile of the Fe I $\lambda 6173 \AA$ line under the asun atmosphere with zero magnetic field. The LOS is assumed to be normal to the surface $\left(\theta=0^{\circ} \rightarrow \mu=\cos (\theta)=1\right)$ and furthermore we have assumed solar iron abundance of $\epsilon_{\mathrm{Fe}}=-4.37$ ( defined as $\log n_{\mathrm{Fe}} / n_{\mathrm{tot}}$ ). In Fig. 3, we show the intensity line profile calculated with both codes (solid black is iMap, dashed red is COSSAM). Both Stokes $I$ profiles are virtually indistinguishable within Fig. 3. The relative rms error (relative to the continuum) between both profiles is less than $0.1 \%$, a value, which is comparable to the inter-agreement study of Wade et al. (2001).

\subsubsection{Local line profiles - kilo Gauss magnetic field}

We now introduce a volume filling homogeneous (depthindependent) magnetic field of $1 \mathrm{kG}$. The inclination of the field (relative to the LOS) is set to $40^{\circ}$ and the azimuth to $0^{\circ}$. All other parameters are the same as in the preceding section. Figure 4 again demonstrates the remarkable good agreement between the two codes for all Stokes parameter profiles. The rms errors are as follows: for the Stokes $I$ profiles $=0.18 \%$; for Stokes $V=0.4 \%$; for Stokes $Q=1.0 \%$; and for Stokes $U=0.98 \%$. The rms values are given relative to the full amplitude (see Wade et al. 2001) and are well within the margins of their interagreement study.

From these test calculations, we can already state that both synthesis codes show a very good agreement, which is comparable to the inter-agreement between the three synthesis codes INVERS10, ZEEMAN2, and COSSAM.

\section{The artificial neural network approach}

The basic idea of the approach is to emulate the process of polarized line formation by using an adaptive model, that is fast to evaluate, and which provides the required accuracy. The adaptive model we seek must have a sufficient complexity to describe the non-linear mapping of Eq. (1), between the most prominent atmospheric input parameters and the resulting Stokes spectra. For this purpose, we propose a supervised machine learning algorithm, e.g., an artificial neural network (ANN) model. Successful applications of ANNs in the field of spectral analysis and Stokes profile inversions have been presented by Carroll \& Staude (2001, 2003); Socas-Navarro (2005b); Carroll $\&$ Kopf (2008). In this section, we want to show how a popular type of ANN, known as multilayer perceptrons (MLPs), can be used for the forward modeling approach, i.e., the polarized spectral line synthesis. The MLP can be regarded as a class of nonlinear function, which performs a multivariate mapping between some input vector $\boldsymbol{x}$ and an output vector $\boldsymbol{y}$. The network function represents a function composition of elementary non-linear functions $g(a)$. These elementary functions are arranged in layers whereby each of these functions in one layer is connected via an adaptive weight vector $\boldsymbol{w}$ to all elementary functions in the neighboring layers. The $l$ th output (component of the output vector $\boldsymbol{y}$ ) of a three layer (of weights) MLP for example can be written as

$\boldsymbol{y}_{l}(\boldsymbol{x} ; \boldsymbol{w})=g_{l}\left(\sum_{k=0}^{K} w_{l k}^{(3)} g_{k}\left(\sum_{j=0}^{J} w_{k j}^{(2)} g_{j}\left(\sum_{i=0}^{I} w_{j i}^{(1)} x_{i}\right)\right)\right)$,

where $x_{i}$ represents the $i$ th component of the input vector $\boldsymbol{x}$ and $w_{j i}^{(1)}$ the connecting weight from the ith input component to the $j$ th elementary function $g_{j}$ in the first unit layer. The weights $w_{k j}^{(2)}$ then connecting all the functions $g_{j}$ with the functions $g_{k}$ in the second unit layer and $w_{l k}^{(3)}$ finally connects all functions $g_{k}$ from the second unit layer to the functions $g_{l}$ in the third unit layer. The capital letters $(I, J, K)$ giving the numbers of elementary functions (units) in the respective unit layer. The elementary functions $g(a)$, which are also called activation functions, are given by the following type of sigmoid function,

$g(a)=\frac{1}{1+\exp (-a)}$

The network function $\boldsymbol{y}(\boldsymbol{x})$ will thus process a given input vector $\boldsymbol{x}$ by propagating this vector (by multiplication with the individual weight values and subsequent summation and evaluation of the different activation functions) through each layer of the network.

The particular function that will be implemented by the MLP is determined by the overall structure of the network and the individual adaptive weight values. The process of determining these weight values for the network function (MLP) is called (supervised) training and is formulated as a non-linear optimization problem. This training is performed on the basis of a representative dataset, which includes the input to target relations of the underlying problem. This process is similar to a non-linear regression for a given data set, but as the underlying model (i.e., MLP) is much more general, the regression function is not restricted to a specific predetermined (or anticipated) model. In fact, it can be shown that a MLP provides a general framework for approximating arbitrary non-linear functions (Bishop 1995). As for all statistical learning methods particular attention must be given to the dataset used for the training, which should contain a representative and statistical relevant sample of the underlying function we wish to approximate. In our case the input-to-target relation is dictated by our synthesis problem and therefore the input vectors are given by the underlying atmospheric parameters (magnetic field, temperature etc.) and the target vectors, which 

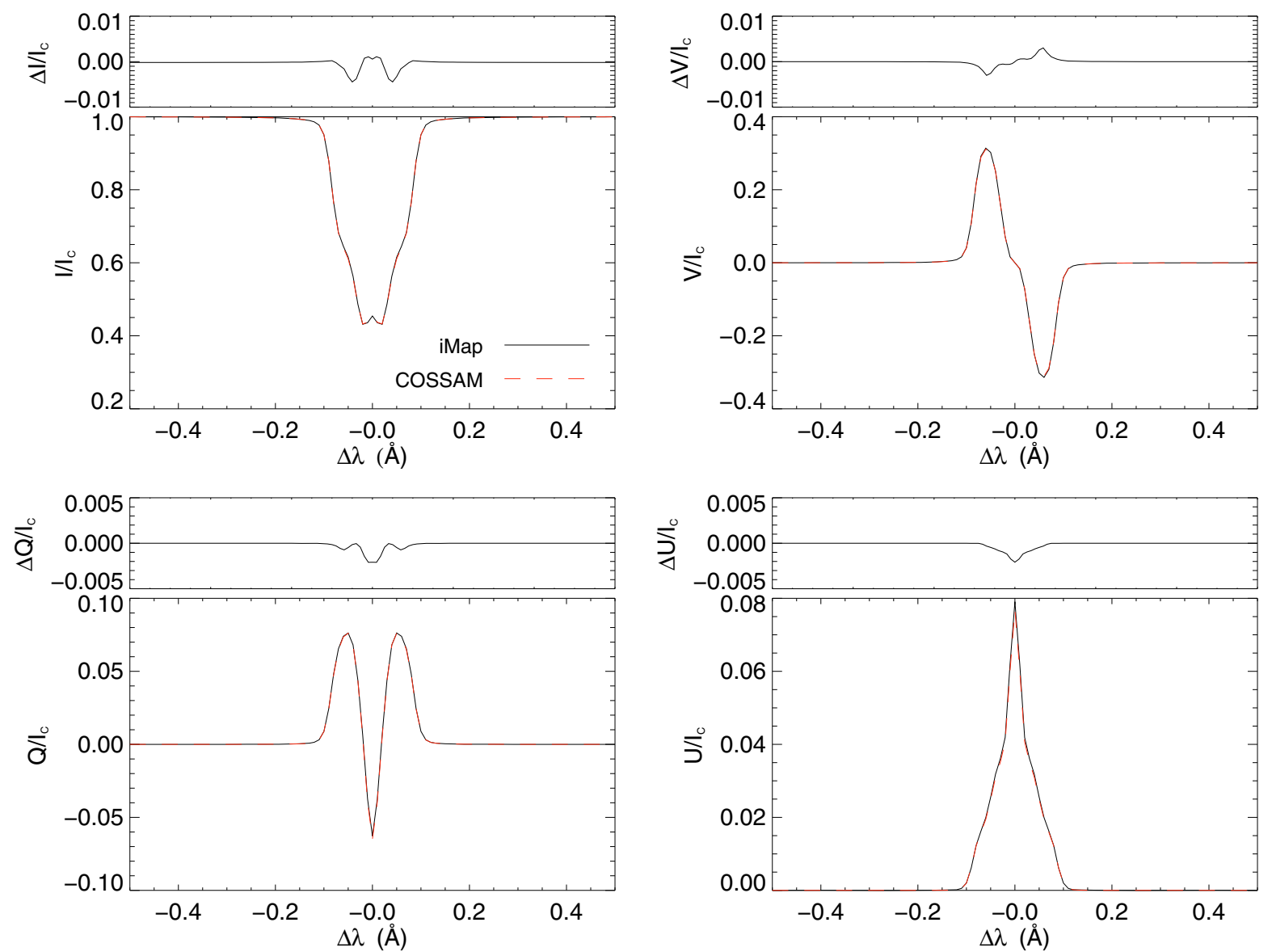

Fig. 4. Comparison of Stokes I profiles (top, left), Stokes $V$ (top, right), Stokes $Q$ (bottom, left) and Stokes $U$ (bottom, right) for the $1 \mathrm{kG}$ case. Calculations made with iMap are again in solid black lines and those made with COSSAM in dashed red lines. Difference plots are shown again on top of each profile plot.

result from the polarized radiative transfer, by the corresponding profiles of the Stokes spectra. The training process (optimization) then tries to adjust the free parameters (i.e. weights) of the network to find an approximation for the underlying generator (function) of the training data. The optimization problem is formulated as a non-linear, least-square problem and is described by the following summed-square error function

$E=\frac{1}{2} \sum_{n=1}^{N} \sum_{k=1}^{C}\left\{y_{k}\left(\boldsymbol{x}^{n} ; \boldsymbol{w}\right)-t_{k}^{n}\right\}^{2}$,

where $N$ is the number of training patterns (i.e., pairs of inputtarget vectors) in the training set and $\mathrm{C}$ the number of output units (elements) of the network; $y_{k}\left(\boldsymbol{x}^{n} ; \boldsymbol{w}\right)$ the network function for the $k$ th output component given the $n$th input vector $\boldsymbol{x}^{n}$ of the training sample. The value $t_{k}^{n}$ is the $k$ th (target) component of the $n$th training vector.

The optimization of the network function is then performed by a non-linear least-square procedure. Once the network is successfully trained and has converged in terms of minimizing the error function Eq. (9) for the training samples, the network weights are frozen and the MLP, which now represents the desired approximation of the underlying function, can be applied to new and unknown data coming from the same parameter domain as the training data.

\subsection{Synthesis of the Stokes database and principal component analysis}

To properly train the network function Eq. (7), we have to generate an exhaustive training database of synthetic Stokes spectra. For the task of building the training database, we have calculated with our ZDI code iMap a large database of synthetic local Stokes spectra. To provide the network with the necessary information about the underlying mapping, we have identified the following atmospheric quantities as input parameters for the MLP: the temperature and pressure structure of model atmospheres (described by the effective temperature $T_{\text {eff }}$ ), gravitation $(\log g)$, iron abundance $\left(\epsilon_{\mathrm{Fe}}\right)$, the local bulk velocity $\left(v_{\text {bulk }}\right)$ of the plasma, microturbulence $\left(v_{\text {mic }}\right)$, macroturbulence $\left(v_{\mathrm{mac}}\right)$, magnetic field strength $\left(B_{\mathrm{loc}}\right)$, magnetic field inclination $\left(\gamma_{\mathrm{loc}}\right)$, magnetic field azimuth $\left(\chi_{\text {loc }}\right)$ and the LOS angle between observer and the local normal $\left(\theta_{\mathrm{loc}}\right)$ of the surface element. The latter parameter determines also the center-to-limb variation (limb darkening) of the disk-integrated spectra. The magnetic field parameters are assumed to be depth independent.

To confine the possible combination of the input parameters, but still provide a reasonable flexibility over a wide range of parameters for the MLP spectrum synthesis, we divided the atmospheric quantities in a set of variable and static (fixed) parameters. The static parameters are initially chosen and then fixed during the course of the investigation. The variable as well as static parameters and their associated parameter ranges are listed in Table 1. 
Table 1. Variable and static parameters and their corresponding parameter ranges.

\begin{tabular}{ccc}
\hline \hline Lower value & Variable parameter & Upper value \\
\hline $4500 \mathrm{~K}$ & $T_{\text {eff }}$ & $6500 \mathrm{~K}$ \\
$0 \mathrm{G}$ & $B_{\text {loc }}$ & $2500 \mathrm{G}$ \\
$0^{\circ}$ & $\gamma_{\text {loc }}$ & $180^{\circ}$ \\
$0^{\circ}$ & $\chi_{\text {loc }}$ & $180^{\circ}$ \\
$0^{\circ}$ & $\theta_{\text {loc }}$ & $90^{\circ}$ \\
\hline Value & Static parameter \\
\hline-4.37 & $\epsilon_{\mathrm{Fe}}$ \\
4.0 & $\log (g)$ & \\
$2.0 \mathrm{~km} \mathrm{~s}^{-1}$ & $v_{\text {mic }}$ & \\
$2.0 \mathrm{~km} \mathrm{~s}^{-1}$ & $v_{\text {mac }}$ & \\
$0.0 \mathrm{~km} \mathrm{~s}^{-1}$ & $v_{\text {bulk }}$ & \\
\hline
\end{tabular}

The spectral line we have synthesized is the iron line Fe I $\lambda 6173 \AA$, which exhibit a normal Zeeman triplet and is a suitable line for the Zeeman diagnostic because of its large effective Landé factor $\left(g_{\text {eff }}=2.5\right)$ and because this line experiences only minor contribution from surrounding blends. For each Stokes parameter $(I, Q, U, V)$ we have calculated 20000 Stokes spectra by choosing random values for the individual input parameters from the listed parameter ranges (Table 1). The static parameters also enter into the calculation with the values given in Table 1, but were not modified. Using a random generator with a uniform distribution ensures moreover that the available parameter space was evenly sampled. Note that, even though the 5-dimensional parameter space for the variable input parameters is relatively sparsely sampled, MLPs and many ANNs in general are much less susceptible to what is described as the curse of dimensionality (the exponential growth of hypervolume) than conventional parameterized methods (Bishop 1995).

The spectral lines were calculated in a wavelength range of $\pm 1.0 \AA$, around the central wavelength, the spectral resolution for the synthesis was $0.01 \AA$, which results in a total of 201 wavelength points. Since the intrinsic dimensionality of the Stokes profiles is usually much lower than the typical wavelength sampling, the individual line profiles can be described in a much more compact and reduced form than in the original wavelength domain (Asensio Ramos et al. 2007). Taking advantage of the extensive training database, we have applied a principle component analysis (PCA) to decompose the individual Stokes profiles $\boldsymbol{x}_{n}$, by calculating the respective covariance matrix $\boldsymbol{C}_{x}$ of our training database,

$\boldsymbol{C}_{\boldsymbol{x}}=\sum_{n}\left(\boldsymbol{x}_{n}(\lambda)-\overline{\boldsymbol{x}}(\lambda)\right)\left(\boldsymbol{x}_{n}(\lambda)-\overline{\boldsymbol{x}}(\lambda)\right)^{T}$,

where $\lambda$ is the wavelength, $n$ the number of individual spectral line profiles used for the analysis, and $\overline{\boldsymbol{x}}$ the mean Stokes profile of all spectral lines. A new set of coordinate axes can then be determined by calculating the eigenvectors $\boldsymbol{s}_{i}$ and eigenvalues $v_{i}$ of the covariance matrix Eq. (10),

$\boldsymbol{C}_{\boldsymbol{x}} \boldsymbol{s}_{i}=v_{i} \boldsymbol{s}_{i}$

In the PCA analysis, the eigenvectors $s_{i}$ are grouped in descending order according tho their eigenvalues $v_{i}$ which account for the degree of variance in the data (Stokes spectra). The Stokes spectra of the database can then be described by the orthonormal set of eigenvectors,

$\boldsymbol{x}_{k}(\lambda)=\sum_{l} \alpha_{k, l} \boldsymbol{s}_{l}(\lambda)$ where $\alpha_{k, l}=\boldsymbol{x}_{k}(\lambda) \boldsymbol{s}_{l}(\lambda)$ is the scalar product (projection coefficient) between the Stokes profile $\boldsymbol{x}_{k}(v)$ and the eigenvector $\boldsymbol{s}_{l}(\lambda)$. Retaining only the first few dominant eigenvectors (the principal components), which account for the majority of the variance in the data, we find an efficient way of reducing the dimensionality of the Stokes spectra by using only the projection coefficients of the principal components. The representation of the $k$ th original spectra $\boldsymbol{x}_{k}$ can then be expressed with the first $l$ eigenvectors as

$\boldsymbol{x}_{k}(\lambda)=\sum_{m \leq l} \alpha_{k, m} \boldsymbol{s}_{m}(\lambda)$.

The projection coefficients $\alpha_{k, m}$ provide the reduced (decomposed) representation of the original $k$ th profile. With this technique we have reduced the dimension of each spectra from 201 wavelength points to 9 for Stokes $I$ and $V$ and 14 for Stokes $Q$ and $U$. The rms reconstruction error for the PCA decomposed spectra is in all cases less then $0.1 \%$.

\subsection{Training of the MLP synthesis networks}

For the task of approximating the polarized line formation process, according to Eq. (1) we divided the approximation of the Stokes profiles into 5 individual MLP network models. For each Stokes parameter $(I, Q, U, V)$ we use a single MLP that calculates the normalized line profiles (relative to the continuum intensity), and one MLP network that calculates the continuum intensity on top of the atmosphere at the central wavelength of the respective spectral line. The continuum intensity is expressed in units of $10^{6} \mathrm{erg} \mathrm{cm}^{-2} \mathrm{~s}^{-1} \mathrm{sr}^{-1} \AA^{-1}$. Even though we could have used one MLP model for the entire process of calculating the profiles of the complete Stokes vector, we decided to split up the networks to avoid saturation effects during the training process, which may be caused by the differences in the resulting magnitude of the individual Stokes parameter as well as by the different sensitivities (responses) to the atmospheric input parameter.

The 5 MLP network structures are implemented with three layers of weights according to Eq. (7). The input and output structure of the network is dictated by the problem. In our case, each synthesis network has as much input unit (elements) as there are variable parameters (Table 1), i.e., the effective temperature of the model atmosphere, the magnetic field strength, the inclination of the magnetic field, azimuth of the field and the LOS between the surface normal and the observer. The static parameters were kept fixed to their values listed in Table 1 and do not appear explicitly in the network structure. The output structure of the network is given by the decomposed representation of the Stokes profiles. Altogether we end up with two MLP synthesis networks (for Stokes $I$ and $V$ ) that have 5 input parameters and 9 output parameters (the PCA decomposed line profiles), two MLP synthesis networks (for Stokes $Q$ and $U$ ) with 5 input and 14 output parameters (the PCA decomposed line profiles for $Q$ and $U$ ), and one MLP synthesis network for the continuum calculation, which has 5 input parameters and one output for the continuum intensity.

Prior to the training process, the network inputs (atmospheric parameters) as well as the outputs (decomposed spectra) of the training database were rescaled. A process that is necessary for the input parameters due to their different magnitudes, and also for the output parameters due to the limited output range $([0,1])$ of the sigmoid function (Eq. (8)) in the output layer of the MLP networks, note that the input range is $(-\infty,+\infty)$. Each individual input parameter was rescaled such that all rescaled parameters have zero mean and a unit standard deviation. The output values were rescaled into the linear slope [0.1,0.9] of the 

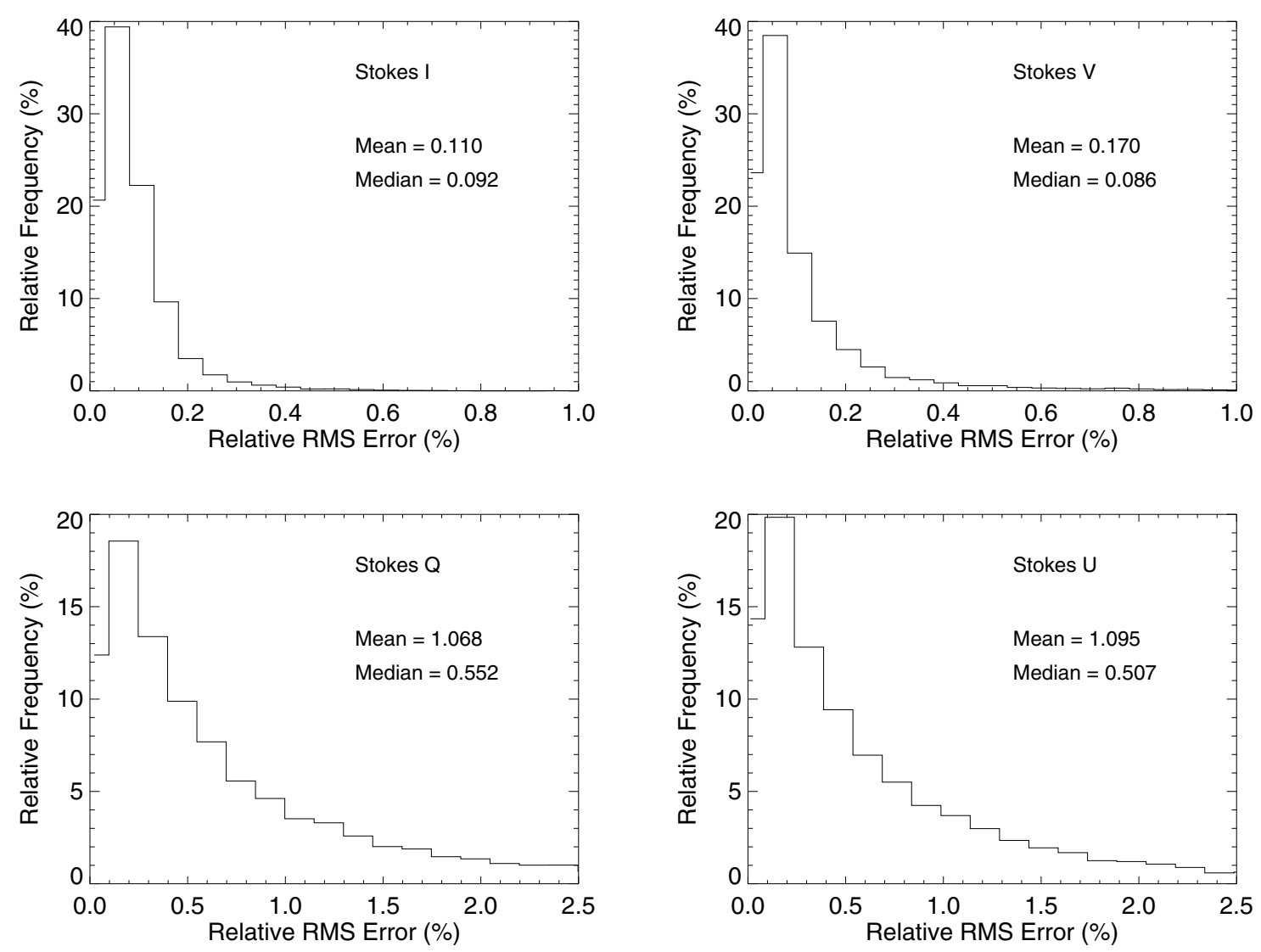

Fig. 5. The rms error distribution between the true numerical- and the MLP-calculated Stokes profiles. The validation sample consists of 13000 profiles for each Stokes parameter. Due to the asymmetry of the distribution, we also list the median besides the mean values. The mean error for Stokes $I$ (top left) is $0.11 \%$, for Stokes $V$ (top right) $0.17 \%$, and $1 \%$ for Stokes $Q$ (bottom left) and Stokes $U$ (bottom right). Note that the median values for Stokes $Q$ and Stokes $U$ are significantly lower than the mean values.

sigmoid function. The 5 synthesis networks were then trained on the basis of the synthetic database (20000 Stokes profiles for each Stokes parameter) to obtain an approximate model for the non-linear mapping between the atmospheric input parameters and the Stokes spectra. The effective complexity of the network and overfitting is controlled during the training process by an early stopping technique (Bishop 1995). Overfitting is here indicated by the divergence of the network error between the training dataset and an independent validation dataset. For the training process and the optimization of the MLP networks we have used a conjugate gradient algorithm (see details Carroll \& Staude 2001). After experimentally testing with different network topologies, in terms of numbers of hidden layers and units therein, we found a sufficiently good convergence and reduction of the squared error for the following MLPs with three weight layers (two hidden unit layers): Stokes $I$ and $V$ 5-50-35-9, Stokes $Q$ and $U$ 5-55-55-14.

After a successful convergence of the training process, the network weights of the individual MLPs are frozen and the networks now (in application mode) represent the desired approximation of the spectrum synthesis function for the iron line Fe I $\lambda 6173 \AA$.

\section{Results}

To evaluate the performance of the trained MLPs, we have created another independent validation database of 13000 synthetic local Stokes profiles for each Stokes parameter (52 000 in total) with our iMap code. The combinations of the input parameters are again determined by a random generator from the parameter ranges listed in Table 1. The calculated Stokes spectra are then decomposed by the PCA technique as described above. To ensure a valid decomposition, the new validation spectra are decomposed on the basis of the training database. Also, for the final input scaling process, the mean and standard deviations from the training database are used. To assess the accuracy of the MLP calculation we compare the results of the MLP network calculation with the real Stokes profiles numerically calculated with iMap using the DELO integration method. Prior to the comparison, the calculated (scaled) PCA profiles from the MLP were rescaled and converted (reconstructed) to the wavelength domain.

\subsection{Local Stokes profiles}

The impressive results for the 52000 local test Stokes spectra of the validation set are illustrated in the histograms in Fig. 5. The relative rms error is again calculated relative to the continuum intensity for Stokes $I$ and to the full amplitude for Stokes $V$, $Q$, and $U$. The mean rms error for the Stokes $I$ profile calculation with the MLP is as low as $0.11 \%$; for Stokes $V, 0.17 \%$; and for Stokes $Q$ and $U$, slightly above $1 \%$. Note that due to the pronounced asymmetry of the Stokes $Q$ and $U$ error distribution the median of the distribution is only $0.5 \%$. The reason 


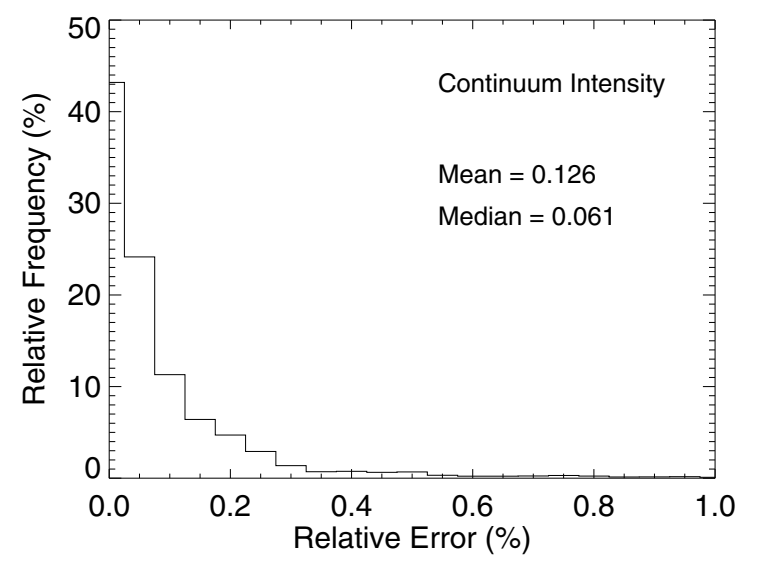

Fig. 6. The distribution of the relative discrepancy between the numerically-calculated continuum intensity and the MLP calculation. The mean rms error is $0.126 \%$ and the median $0.061 \%$ which show that the MLP is able to approximate the calculation of the continuum intensity with high accuracy.

why the synthesis of the Stokes $Q$ and Stokes $U$ profiles are systematically larger than those for Stokes $I$ and $V$ is caused mainly by two effects, first, for relatively weak fields (and thus small profiles) Stokes $Q$ and Stokes $U$ are second order effects (Landi degl'Innocenti 1992) which results in increased relative error contribution, and second, both components periodically drop to zero for certain azimuthal and inclination values, which again leads to very small resulting profiles and hence to an increase in the relative error (see also discussion below). Figure 6 shows the distribution of the relative error compared with the numerical calculated value of the continuum intensity with iMap, which also show a very good agreement.

Even though effects like the temperature variation and magnetic field variation leave their characteristic imprint in the Stokes spectra, such that an ANN is able to approximate this behavior, it should be kept in mind however that this will be done in a complex and non-linear fashion by the transport of polarized radiation. In this way, the approximation capabilities and accuracy of the trained MLPs are quite remarkable. The MLPs have clearly been able to disentangle the effects of the different variable input parameter to calculate the correct Stokes spectra. Note that for the Stokes profiles as well as for the continuum synthesis, the effects of the LOS angle $\theta$, which leads to a variation of the temperature and pressure stratification over the optical depth scale, and which is responsible for the limb darkening effect, could apparently be well distinguished by the synthesis networks from other competing effects like the magnetic field strength, inclination, and the effective temperature of the model atmosphere.

To display how the errors are distributed over the main input parameter, we show in Fig. 7 the relative rms errors over four atmospheric parameters. For the Stokes $I$ profiles (Fig. 7, top row) we obtain a relative homogeneous distribution for the effective temperature (top row, first column) as well as for the magnetic field azimuth (top row, fourth column) which, of course, plays hardly any role for the Stokes I profile. The situation is different for the magnetic field inclination (top row third column), although the rms error is quite low throughout the field inclination regime $\left(0^{\circ}\right.$ to $\left.180^{\circ}\right)$ one notice the increase toward the longitudinal orientation of the field (close to $0^{\circ}$ and $180^{\circ}$ ). This can be explained by the quasi bias (border effects) in the uniformly distributed training database where the pure longitudinal effect with a vanishing pi-component is underrepresented in the database and the more common non-longitudinal effect (elliptical polarization) with three contributions (two sigma- and one pi-components) is far more common. Nevertheless, note the remarkable agreement through the entire range. For the Stokes $I$ error over the magnetic field strength (top row, second column) we get a somewhat counterintuitive result, which shows an increase of the error with field strength. One would assume a decrease of the error since for increasing field strength the individual Zeeman components are increasingly separated. For strong enough fields, such that the separation of the individual Zeeman components is well beyond the spectral line width, one would assume that the linear dependence of the Zeeman separation upon the field strength should be recognizable for the MLP and therefore the functional behavior should be even easier to learn for the MLP. This is in principle true but the PCA decomposition of the spectra offsets this effect and acts in the opposite direction. The decomposition of a completely Zeeman-splitted Stokes $I$ profile on the basis of the current dataset requires a stronger representation of the higher-order (i.e., less significant) eigenprofiles to describe the entire profile correctly. This means that the projection coefficients corresponding to these higher-order eigenprofiles have a significant value. Despite the simpler functional dependence in the Zeeman saturated regime, the PCA decomposed Stokes vector gets more complicated due to the nonnegligible, higher-order projection coefficients. This effect could be easily diminished by using different datasets for the weakfield and the strong Zeeman saturated regime. However, note that the maximum error for strong fields is still remarkable low (lower than $0.22 \%$ ) and we therefore refrain from splitting the database in this study.

For the Stokes $V$ profiles (Fig. 7, second row) we also get very satisfying results. Over the entire temperature range (second row, first column) we see a low error, which is around the total mean value of $0.17 \%$. The slight peak around 5500 and $6000 \mathrm{~K}$ can be explained by the coincidental accumulation of low inclination angles in that region. For the rms error over the field strength (second row, second column) we notice a slight systematic increase for smaller field strength. This can be explained by the smaller amplitude of the Stokes $V$ profiles in that range which are harder to disentangle from other effects like temperature and inclination. For higher field strengths, the Stokes $V$ profiles get increasingly separated, which again affects the PCA representation of these profiles and thus the reconstruction error (see above). The overall error for the magnetic field strength is also very close to the total mean of $0.17 \%$. As the Stokes $V$ signals are almost independent of the azimuthal angle (except for small magneto-optical effects), we see no significant variation along the azimuthal angle (second row, fourth column). For the inclination (second row, third column), we see a rise in the error for angles around $90^{\circ}$ which displays the $\cos (\gamma)$ dependence of the Stokes $V$ profile on the inclination angle (Eq. (6)), which leads to a rapid decrease to around $90^{\circ}$. Although the absolute error is not much affected by the decrease of the signal to around $90^{\circ}$, the relative error increases for very small signals. However, note that the rms error is even for the very weak signals around $90^{\circ}$ lower than $0.7 \%$.

For Stokes $Q$ and $U$, we obtain similar good results (Fig. 7 third and fourth row). In both cases, we see a relative homogeneous error distribution over the temperature (first column) as well as for the field strength (second column). The error distribution over the inclination (third column) shows a relatively strong increase toward $0^{\circ}$ and $180^{\circ}$. This increase can be understood by the smallness of the resultant Stokes $Q$ and $U$ profiles for 

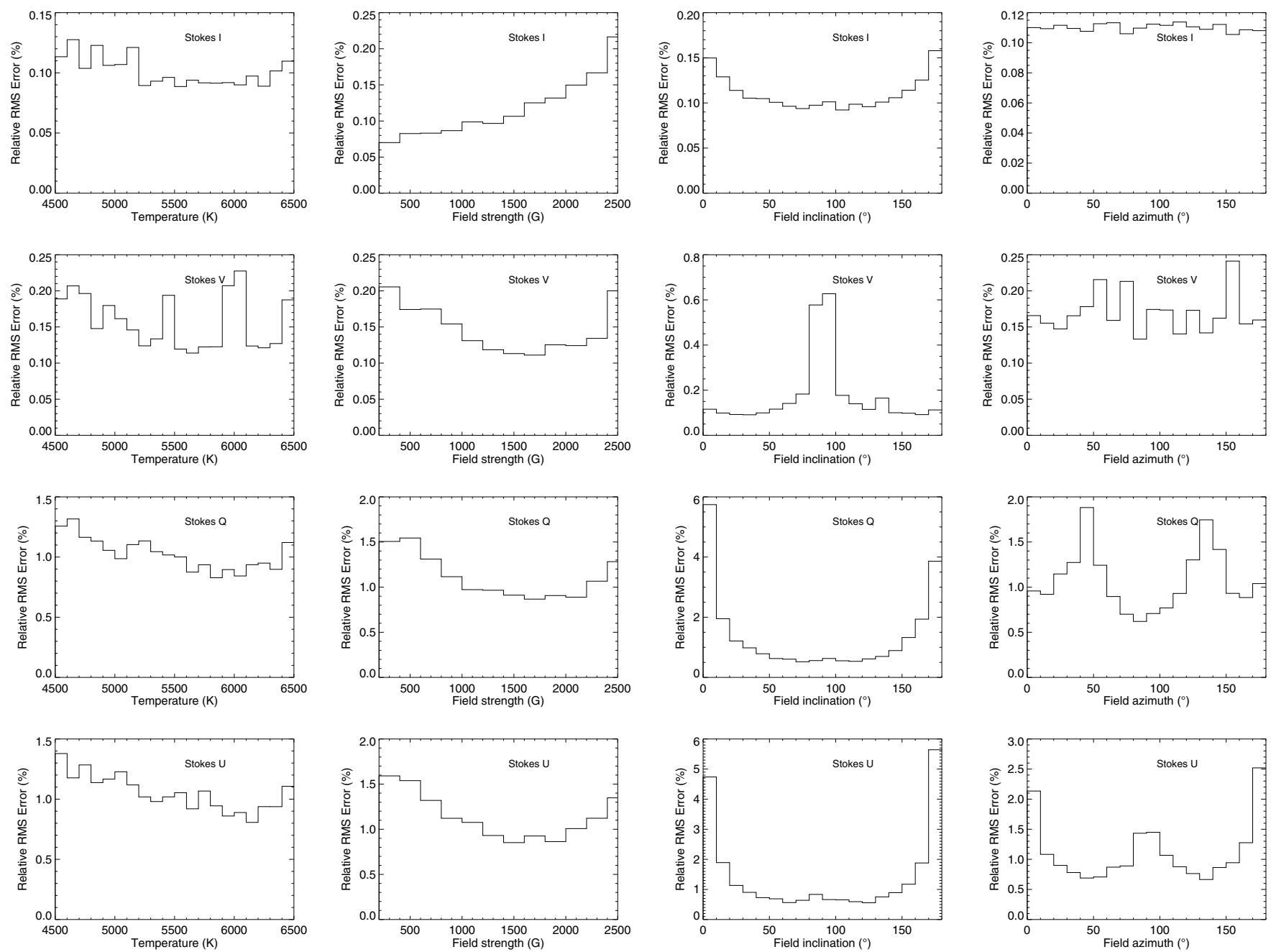

Fig. 7. The distribution of the rms errors for individual atmospheric parameters. In each row, the distributions for one Stokes parameter are listed: (Stokes I, first row; Stokes $V$, second row; Stokes $Q$, third row; Stokes $U$, fourth row). In each column, the distributions for a particular atmospheric parameters are given: temperature (1st column), field strength (2nd column), field inclination ( 3 rd column), and the field azimuth (4th column).

these inclination angles and the associated increase in the relative error. But note that even for strongly inclined fields the MLP synthesis yields a rms error that is smaller than $6 \%$. The distribution reflects here the $\cos (2 \chi)$ and $\sin (2 \chi)$ dependence (see Eq. (6)) on the azimuthal angle, which leads to a rapid decrease of the Stokes signal at these angles and therefore again to an increase of the relative error. These two effects in conjunction (inclination and azimuth) are the main causes for the larger relative error of the Stokes $Q$ and $U$ synthesis networks compared to the Stokes $I$ and $V$ networks. But nevertheless both synthesis networks (Stokes $Q$ and $U$ ) have learned to approximate the complicated relation and perform remarkably well over the entire range of parameters.

To obtain yet another impression about the accuracy, we have compiled a short list of representative Stokes $I, V, Q$, and $U$ profiles in Fig. 8 calculated under various combinations for the LOS angle of the atmosphere $\theta$, the effective temperature of the model atmosphere $T_{\text {eff }}$, magnetic field strength $|B|$, the magnetic field inclination $\gamma$ and the magnetic field azimuth $\chi$. Each row in Fig. 8 shows the set of four Stokes profiles calculated under a specific atmospheric conditions. The atmospheric parameters are specified for each row in the Stokes I plot (first column) The profiles obtained by the full numerical solution (denoted by DELO) are shown in solid black lines, while the MLP calculations are shown in dashed red lines. For all conditions (set of free parameters) the plots show a very good agreement between the full numerical solution and the MLP calculation.

\subsection{Disk integrated Stokes profiles}

To evaluate the accumulated effect of a disk-integrated spectrum we have also calculated the resulting profiles for two synthetic test star models within the iMap code. The first model consists of a potential field where the individual contributions to the magnetic field vector are described by spherical harmonics (Altschuler \& Newkirk 1969). For the Legendre parameter, we have chosen $l=4$ and $m=3$, which account for a sufficient complex surface distribution of the field strength, inclination, and azimuth. For the magnetic field strength, we have assumed a peak value of $1400 \mathrm{G}$. The surface distribution for the radial field component is shown in Fig. 9.

For the synthesis we used a rotational velocity with a $v \sin i$ of $26 \mathrm{~km} \mathrm{~s}^{-1}$. Furthermore, we again used the iron line Fe I $\lambda 6173 \AA$ and a Kurucz model atmosphere $(\log g=4.0)$ with an effective temperature of $5000 \mathrm{~K}$. The static parameters are those listed in Table 1. The synthetic spectra are calculated 

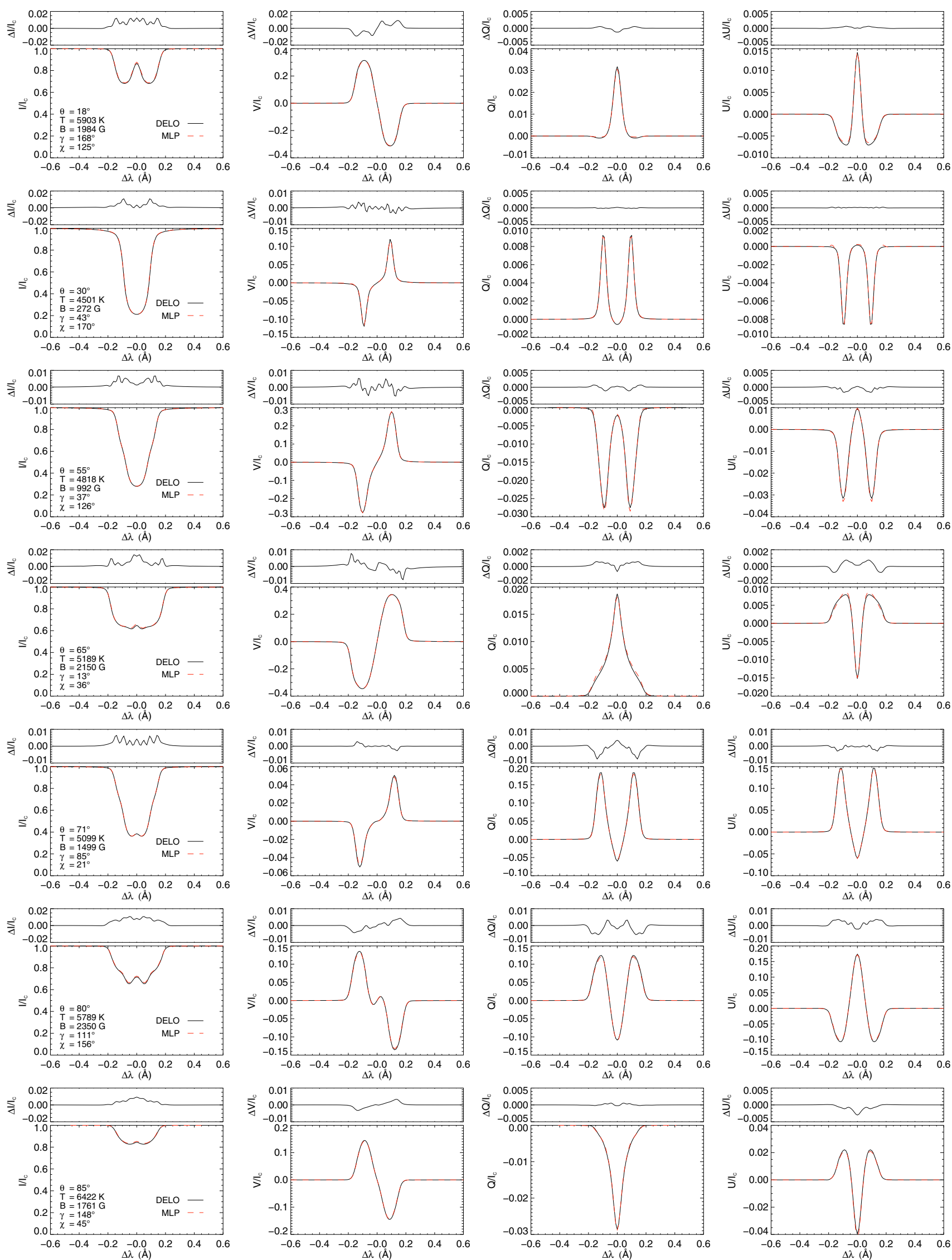

Fig. 8. Each row shows the set of the four Stokes profiles $(I, V, Q, U)$, synthesized for a particular realization of the free parameters. The particular values of the free parameters are always specified in the Stokes I plot (first column). Profiles calculated with the full numerical solution (denoted by DELO), are drawn with solid black lines, while the profiles calculated with our MLP method shown in dashed red lines. Difference plots for each Stokes parameter are provided again on top of each profile plot. 


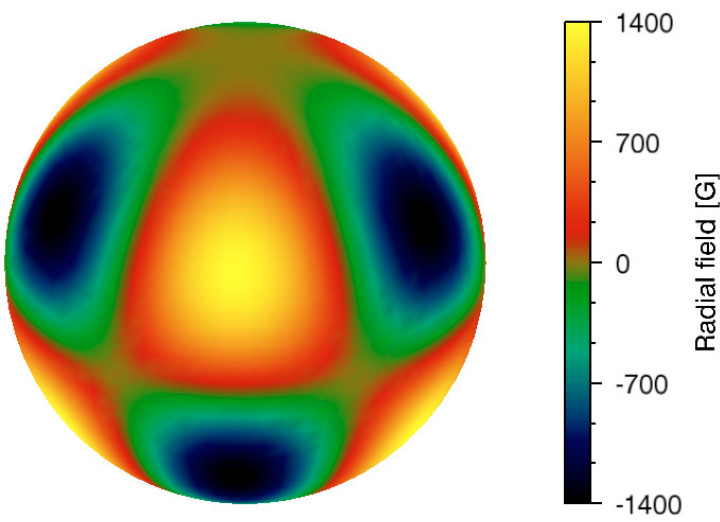

Fig. 9. The radial component of the surface field for the spherical harmonic test star, with $l=4, m=3$. The effective temperature of the atmospheric model is $5000 \mathrm{~K}$ and the peak field strength is $\pm 1400 \mathrm{G}$.

with a surface resolution of $3 \times 3$ degree, which results, for the visible hemisphere, in 3600 surface elements. For each of these surface elements the underlying model atmosphere is adjusted with respect to the local LOS angle $\theta$ to correctly model the center-to-limb variation (limb darkening).

On the basis of the stellar model, the disk-integrated spectrum is calculated within iMap, one run with the full numerical solution and another with the MLP synthesis module. In Fig. 10 the disk-integrated Stokes spectra are shown for the spherical harmonic model. These plots show very good agreement between the numerical solution denoted by DELO and drawn with solid black lines, and the MLP synthesis shown in dashed red lines. No accumulating errors are visible, and the errors are in fact equal or even smaller than the mean errors derived from the statistical evaluation in Sect. 4.1. The rms error is $0.09 \%$ for the Stokes $I$ profile, $0.18 \%$ for the Stokes $V$ profile, $0.36 \%$ for the Stokes $Q$ and $0.72 \%$ for the Stokes $U$ profile. To provide another model and to test the joint effect of a temperature and a magnetic field variation, we construct with iMap a two spot model star. For the quite stellar surface, we used a Kurucz model atmosphere $(\log g=4.0)$ with an effective temperature of $5500 \mathrm{~K}$ and for the two spots a model that is $1000 \mathrm{~K}$ cooler $(4500 \mathrm{~K})$. The maximum field strength of the spots is set to $1500 \mathrm{G}$ and the field orientation is such that the two spots have a radial field of different polarities. The $v \sin i$ is $35 \mathrm{~km} \mathrm{~s}^{-1}$ and the disk integration is performed with a surface resolution of $5 \times 5$ degree. To provide a smooth continuous gradient in the temperature as well as in the magnetic field within the two spots we have also smeared the field and temperature distribution on the surface with a 2-dimensional Gaussian function. Note that in this model the relative weighting of the local profiles is also affected by the temperature variation and the corresponding continuum intensities, which therefore requires an accurate calculation of the individual continuum intensities by the MLP. The model star is shown in Fig. 11 where we have color coded the surface temperature and depicted the field lines within the spots by blue lines for positive and yellow lines for a negative polarity.

The resultant Stokes profiles for the disk integration are shown in Fig. 12, where we can see again an exceptional agreement between the numerical and the neural network calculation. The rms errors are $0.12 \%$ for the Stokes I profile, $0.37 \%$ for the Stokes $V$ profile, $0.44 \%$ for the Stokes $Q$, and $0.43 \%$ for the Stokes $U$ profile.
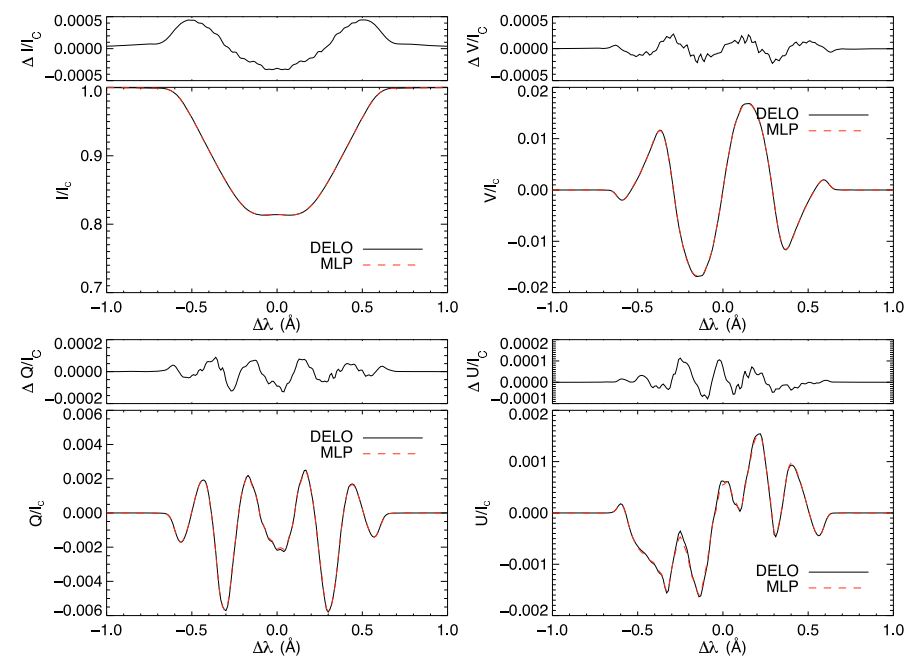

Fig. 10. The disk-integrated Stokes spectra for the spherical harmonic test star. Also, for the disk-integrated Stokes spectra, we see very good agreement between the numerical solution (DELO, solid black line) and the MLP synthesis (dashed red line).

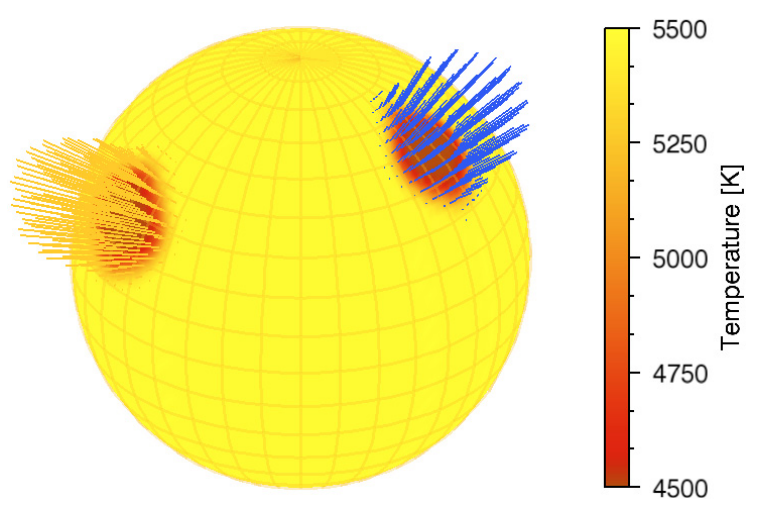

Fig. 11. A two-spot test star with a bipolar spot configuration of $\pm 1500 \mathrm{G}$. The effective temperature for the quiet surface is $5500 \mathrm{~K}$ and $4500 \mathrm{~K}$ for the spots.

\section{Improvement in speed}

One of the main motivations for our proposed approach was of course to find a suitable approximate method which facilitates a fast synthesis of Stokes profiles. Once our MLPs are trained and the network weights are frozen, the network structure represents a simple form of a composite mathematical function (see Sect. 3). The MLP networks can then readily be implemented in a quickly-evaluated programming language or stored on an even faster to evaluate hardware chip (e.g., EPROM). Both approaches (whether software or hardware) have significant potentials to allow for an efficient parallelization. For our purpose we implemented the five trained MLP network structures in the programming language $\mathrm{C}++$ on a single processor desktop computer.

To evaluate the increase in speed provided by our ANN approach, we made the following benchmark test. Both methods, the numerical integration of the polarized radiative transfer equation with DELO (which is one of the fastest numerical methods) and the MLP synthesis with the MLP networks, are used to calculate 10000 times the full set of Stokes profiles $(I, Q, U, V)$ as well as the local continuum intensity. The profiles are calculated in a wavelength range of $\pm 2 \AA$ around the line center with a spectral resolution of $0.01 \AA$, which gives 401 wavelength points in 

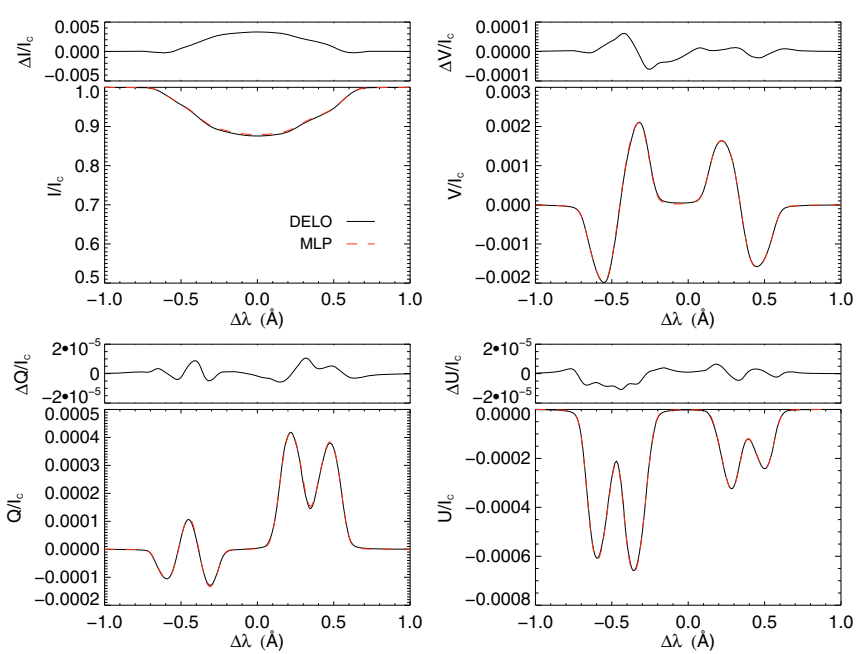

Fig. 12. The disk-integrated Stokes spectra for the two spot test star Again, an impressive agreement between the correct numerical solution (DELO, solid black line) and the MLP synthesis (dashed red line) is achieved.

total for each Stokes parameter and each run. The spectral line is again the iron line Fe I $\lambda 6173 \AA$. The DELO routine integrates the spectral lines within a depth range of $\log \left(\tau_{5000}\right)=1.0$ and $\log \left(\tau_{5000}\right)=-5.0$. The input parameters for the numerical integration and the MLP method are chosen randomly, but prior to the calculation and were kept fixed for the entire benchmark cycle. By this we allow the numerical integration method to calculate and compile the absorption matrix Eqs. (4) and (6), once and for all in advance. Moreover, it avoids the time consuming reading of model atmospheres and line parameters.

The benchmark was performed on several personal computers with varying performance characteristics (processors ranging from $1.7 \mathrm{GHz}$ to $3.2 \mathrm{GHz}$ and RAM from $512 \mathrm{MB}$ to $1 \mathrm{~GB}$ ). The test runs for the ANN/MLP method takes between 2.1 and $4.2 \mathrm{~s}$ while the conventional numerical calculation of the polarized radiative transfer with the DELO method takes between 2820 to $3610 \mathrm{~s}$. This gives an improvement in speed by factors of 860 to 1340 ! It is this significant improvement in speed - by three orders of magnitude - which facilitates a complete disk-integration of a high-resolution stellar model within a few seconds. With this accelerated Stokes profiles synthesis, the complete cycle of forward and inverse calculation for ZDI applications now become possible within a reasonable time frame.

\section{Discussion and conclusions}

In this paper we have introduced, described and evaluated a novel LTE Stokes spectrum synthesis method based on ANNs. Artificial neural networks are used to approximate the LTE polarized radiative transfer by modeling the functional relationship between the most important atmospheric parameters and the corresponding local Stokes spectra. The ANN approach with MLPs is based on an extensive training database of calculated synthetic Stokes profiles. Because of this crucial dependence for the network training we first assessed the accuracy of our polarized radiative transfer module integrated in our ZDI code iMap by a detailed comparison with the existing synthesis code COSSAM in Sect. 3. Both codes exhibit congruent results for several composite values like the continuous absorption coefficient and broadening parameters. Moreover, both codes show a very good agreement in all calculated Stokes profiles. By verifying the agreement between iMap and COSSAM, we also indirectly established an inter-agreement between iMap and INVERSE10 as well as ZEEMAN2, as derived from the inter-agreement study of Wade et al. (2001).

In Sect. 4, we introduced the concept of our proposed method and described the data preparation with a PCA method as well as the training process for the ANNs. A detailed evaluation of our ANN synthesis revealed the remarkable accuracy with which the ANN approach is able to approximate the polarized radiative transfer process. Stokes profiles of the spectral line Fe I $\lambda 6173 \AA$ are calculated with high accuracy for different configurations of the atmospheric input parameters (effective temperature, magnetic field strength, field inclination, field azimuth, and LOS angle). The ANNs have clearly been able to learn the complex non-linear mapping between the atmospheric input parameters and the resulting Stokes profiles. Although we have restricted in this work the number of variable input parameters to 5, there is no principle limitation to extend the number of atmospheric input parameters. Moreover, the limits that we have set for the parameter ranges (see Table 1) are also not a principle restriction of the ANN approach but rather driven by the constraints to cope with the training and evaluation database in this study.

The ANN approach to spectral line synthesis is also not limited to one spectral line, the ANN method can easily be extended to a wider wavelength range to cover several neighboring spectral lines or successive regions of interest in the wavelength domain. Another interesting possibility, offered by the ANN approach is the modeling of co-added line profiles as used for polarized spectral line extraction and reconstruction algorithms like LSD (Donati et al. 1997) or by the PCA method (Carroll et al. 2007; Martínez González et al. 2008). Instead of synthesizing a huge number of individual Stokes profiles to model, the co-added (and therefore noise reduced) mean line profile, an ANN synthesis can be used to directly calculate this co-added line profile. This would offer a vast improvement in speed and accuracy.

It is also important to note that since the ANN approach is based on the accurate numerical modeling of line profiles, blends can be fully accounted for with this approach. Even heavily blended line profile can be accurately synthesized as long as the training database properly accounts for these blends.

One of the main reasons to approximate or even to entirely bypass a full radiative transfer approach in DI or ZDI applications, is the enormous requirement for massively calculating local Stokes profiles during the inversion process. In our benchmark tests, we could show that our ANN approach is three orders of magnitudes faster then the conventional numerical integration scheme. This enormous acceleration and the impressive accuracy makes the ANN synthesis approach a viable and promising alternative for polarized radiative transfer calculations and facilitates a complete radiative transfer driven approach to DI and ZDI as well as for large scale Stokes profile inversions.

Acknowledgements. The authors would like to thank the referee A. Asensio Ramos for his constructive and helpful comments, which helped to improve this manuscript.

\section{References}

Aller, M. F., \& Everett, C. H. M. 1972, ApJ, 172, 447

Altschuler, M. D., \& Newkirk, G. 1969, Sol. Phys., 9, 131

Anstee, S. D., \& O’Mara, B. J. 1995, MNRAS, 276, 859

Asensio Ramos, A., Socas-Navarro, H., López Ariste, A., \& Martínez González, M. J. 2007, ApJ, 660, 1690

Bishop, C. M. 1995, Neural Networks for Pattern Recognition (Oxford University Press) 
Bolton, C. T. 1970, ApJ, 161, 1187

Carroll, T. A., \& Staude, J. 2001, A\&A, 378, 316

Carroll, T. A., \& Staude, J. 2003, AN, 324/4, 392

Carroll, T. A., \& Kopf, M. 2008, A\&A, 481, L37

Carroll, T. A., Kopf, M., Ilyin, I., \& Strassmeier, K. G. 2007, AN, 328, 1043

Donati, J.-F. 1999, MNRAS, 302, 457

Donati, J.-F., \& Brown, S. F. 1997, A\&A, 326, 1135

Donati, J.-F., \& Collier Cameron, A. 1997, MNRAS, 291, 1

Donati, J.-F., Semel, M., Carter, B. D., Rees, D. E., \& Collier Cameron, A. 1997, MNRAS, 291, 658

Donati, J.-F., Collier Cameron, A., Hussain, G. A. J., \& Semel, M. 1999, MNRAS, 302, 437

Donati, J.-F., Cameron, A., Collier, S. M., et al. 2003, MNRAS, 345, 1145

Donati, J.-F., Howarth, I. D., Jardine, M. M., et al. 2006, MNRAS, 370, 629

Hauschildt, P. H., Allard, F., \& Baron, E. 1999, ApJ, 512, 377

Holweger, H., \& Mueller, E. A. 1974, Sol. Phys., 39, 19

Humlicek, J. 1982, J. Quant. Spec. Rad. Trans., 27, 437

Kochukhov, O., Bagnulo, S., Wade, G. A., et al. 2004, A\&A, 414, 613

Kupka, F., Piskunov, N., Ryabchikova, T. A., Stempels, H. C., \& Weiss, W. W. 1999, A\&AS, 138, 119

Kurucz, R. L. 1992, The Stellar Populations of Galaxies, 149, 225

Kurucz, R. L. 1993, IAU Colloq. 138: Peculiar versus Normal Phenomena in A-type and Related Stars, 44, 87

Landi degl'Innocenti, E. 1992, Solar Observations: Techniques and Interpretation, 71

Landstreet, J. D. 1988, ApJ, 326, 967
Lites, B., Casini, R., Garcia, J., \& Socas-Navarro, H. 2007, Mem. Soc. Astron. Ital., 78, 148

Martínez González, M. J., Asensio Ramos, A., Carroll, T. A., et al. 2008, A\&A, in press

Piskunov, N., \& Kochukhov, O. 2002, A\&A, 381, 736

Piskunov, N. E., Kupka, F., Ryabchikova, T. A., Weiss, W. W., \& Jeffery, C. S. 1995, A\&AS, 112, 525

Rees, D. E., \& Murphy, G. A. 1987, Numerical Radiative Transfer, ed. W. Kalkofen (Cambridge University Press), 241

Rees, D. E., Durrant, C. J., \& Murphy, G. A. 1989, ApJ, 339, 1093

Rees, D. E., López Ariste, A., Thatcher, J., \& Semel, M. 2000, A\&A, 355, 759

Rice, J. B., \& Strassmeier, K. G. 2000, A\&AS, 147, 151

Ruiz Cobo, B., \& Del Toro Iniesta, J. C. 1992, ApJ, 398, 375

Socas-Navarro, H. 2005a, ApJ, 620, 517

Socas-Navarro, H. 2005b, ApJ, 621, 545

Socas-Navarro, H., Trujillo Bueno, J., \& Ruiz Cobo, B. 2000, ApJ, 530, 977

Stenflo, J. O. 1994, Ap\&SS Library (Dordrecht, Boston: Kluwer Academic Publishers), 189

Stift, M. J. 2000, A Peculiar Newsletter, 33

Strassmeier, K. G. 2002, AN, 323, 309

Strassmeier, K. G., \& Rice, J. B. 1998, A\&A, 330, 685

Strassmeier, K. G., Hofmann, A., Woche, M. F., et al. 2003, SPIE, 4843, 180

Strassmeier, K. G., Woche, M., Andersen, M., \& Ilyin, I. 2007, SPIE, 328, 627

Wade, G. A., Bagnulo, S., Kochukhov, O., et al. 2001, A\&A, 374, 265

Wittmann, A. 1974, Sol. Phys., 35, 11 